\title{
Theoretical Predictions for the Direct Detection of Supersymmetric Dark Matter
}

\author{
Carlos Muñoz * \\ Departamento de Física Teórica C-XI and Instituto de Física Teórica C-XVI, \\ Universidad Autónoma de Madrid, Cantoblanco, 28049 Madrid, Spain. \\ E-mail: icarlos.munnoz@uam.es
}

ABstract: We compute the neutralino-nucleon cross section in several supersymmetric scenarios, taking into account all kind of experimental and astrophysical constraints. In addition, the constraints that the absence of dangerous charge and colour breaking minima imposes on the parameter space are also considered. This computation is relevant for the theoretical analysis of the direct detection of dark matter in current experiments. We discuss interesting supergravity and superstring scenarios.

\section{Introduction}

Impressive experimental efforts have been carried out since 1987 for the direct detection of dark matter through elastic scattering with nuclei in a detector [i]. In fact, one of the experiments, the DAMA collaboration ${ }^{1}$, has reported data favouring the existence of a Weakly-Interacting-Massive-Particle (WIMP) signal. This signal is compatible with WIMP masses up to $100 \mathrm{GeV}$ and WIMP-nucleon cross sections in the interval $10^{-6}-10^{-5} \mathrm{pb}$, as shown with a dark shaded area in Fig. 11.1.

Notice, however, that this result has been obtained assuming the simple isothermal sphere halo model with a dark-matter density $\rho_{0}=0.3 \mathrm{GeV} \mathrm{cm}^{-3}$ and a MaxwellBoltzmann local velocity distribution for the WIMPs $f(v) \propto e^{-v^{2} / v_{0}^{2}}$, with $v_{0}=220 \mathrm{~km}$ $\mathrm{s}^{-1}$. When uncertainties on the halo model are taking into account, the signal is consistent with a larger region of the parameter space. In particular, in Ref. [2] modifications in the velocity distribution function for different galactic halo models were considered, using in addition the allowed ranges for $v_{0}$ and $\rho_{0}$ in each model. The final result of the analyses is shown in Fig. $\prod_{i}^{1}$ with a light shaded area. One sees that the signal is compatible with

${ }^{*}$ This work was supported in part by the Spanish DGI of the MCyT under Acción Integrada HispanoAlemana HA2002-0117, and under contracts BFM2003-01266 and FPA2003-04597; and the European Union under contract HPRN-CT-2000-00148.

${ }^{1} \mathrm{~A}$ complete list of references for this and the other experiments discussed below can be found in Ref. [1] $\left.{ }_{1}^{1}\right]$. 


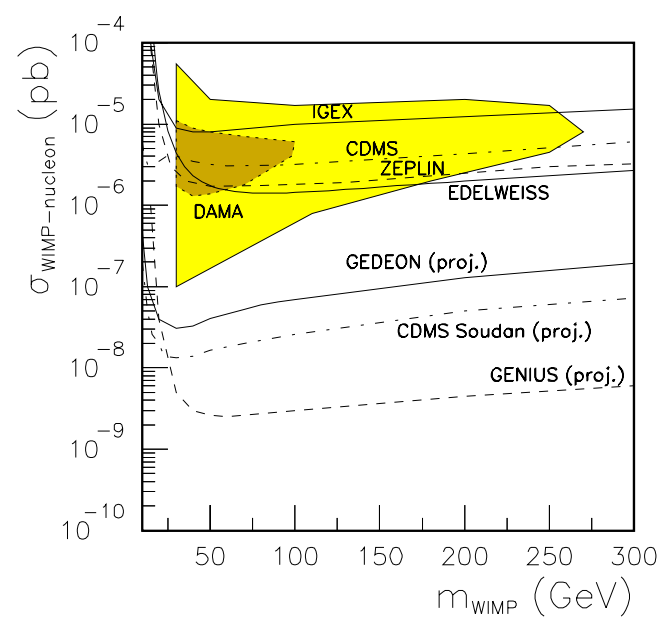

Figure 1: Areas allowed by the different experiments for the direct detection of dark matter in the parameter space $\left(\sigma_{\text {WIMP-nucleon }}, m_{\text {WIMP }}\right)$. The light shaded (yellow) area is allowed by the DAMA experiment, including uncertainties on the halo model. The dark shaded (brown) area inside the previous one corresponds to the simple case of an isothermal sphere halo model. This case is also the one analyzed by the other experiments: The (lower) areas bounded by solid, dot-dashed, dashed and (again) solid lines are allowed by IGEX, CDMS, ZEPLIN I and EDELWEISS current experimental limits. The (upper) areas bounded by solid, dot-dashed and dashed lines will be analyzed by the projected GEDEON, CDMS Soudan and GENIUS experiments.

larger values of the parameters, i.e. WIMP masses up to $270 \mathrm{GeV}$ and WIMP-nucleon cross sections in the interval $10^{-7}-6 \times 10^{-5} \mathrm{pb}$. In fact, as discussed also in Ref. [2], when co-rotation of the galactic halo is also considered, the mass range extends further to $500-900 \mathrm{GeV}$ for cross sections in the interval few $\times 10^{-6}-2 \times 10^{-5} \mathrm{pb}$.

Unlike this spectacular result, other collaborations claim to have excluded important regions of the DAMA parameter space, as shown also in Fig. in. In particular, the first of these was the CDMS experiment A small part of the region excluded by CDMS has also been excluded by IGEX and HDMS. But more disturbing are the recent results from EDELWEISS and ZEPLIN I collaborations, excluding even larger regions than CDMS. Let us remark that, unlike DAMA, for CDMS and for the other experiments analyses taking into account the uncertainties in the galactic halo are not shown in the figure, and we only see the effect of the standard halo model on their results. Including those uncertainties, the light shaded area favoured by DAMA and not excluded by the null searches would be in principle smaller than the one shown here (for an analysis of this issue see Ref. 绿).

Owing to this controversy between DAMA and the other experiments, one cannot be sure whether or not the first direct evidence for the existence of dark matter has already been observed. Fortunately, the complete DAMA region will be tested in the future, since more than 20 experiments are running or in preparation around the world. This is for example the case of GEDEON, an expansion of CDMS called CDMS Soudan, and GENIUS, shown in Fig.

Given this situation, and assuming that the dark matter is a WIMP, it is natural to 
wonder how big the cross section for its direct detection can be. The answer to this question depends on the particular WIMP considered. The leading candidate in this class is

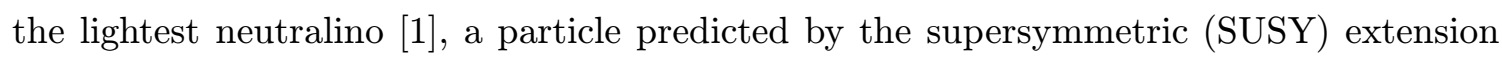
of the standard model. It is then crucial to re-analyze the compatibility of the neutralino as a dark matter candidate, with the sensitivity of present (and future) dark matter detectors. Here we will concentrate on the most recent results in the context of supergravity (SUGRA) and superstrings. Concerning the latter, let us recall that the neutralino is also a candidate for dark matter in superstring constructions, since generically the low-energy limit of superstring theory is 4-dimensional SUGRA.

Let us finally remark that we will impose in our computation the usual experimental and astrophysical constraints in this type of analysis, namely the lower bound on the Higgs mass, the $b \rightarrow s \gamma$ branching ratio, the muon $g_{\mu}-2$, and the observed dark matter density of the Universe. Likewise, the constraints that the absence of dangerous charge and colour breaking minima imposes on the parameter space will also be taken into account $\left[\begin{array}{l}{[\overline{4}} \\ i\end{array}\right]$.

\section{SUGRA predictions}

In this section we will analyse the minimal-supersymmetric-standard-model (MSSM) scenario in the framework of SUGRA. In this framework a large number of free parameters are present in general. In order to have predictive power one usually assumes that the soft parameters are universal at $M_{G U T} \approx 2 \times 10^{16} \mathrm{GeV}$. This is the so-called minimal supergravity (mSUGRA) scenario, where there are only four free parameters: $m, M, A$, and $\tan \beta$. In addition, the sign of $\mu$ remains also undetermined since only $\mu^{2}$ is determined by the minimization of the Higgs effective potential

$$
\mu^{2}=\frac{m_{H_{d}}^{2}-m_{H_{u}}^{2} \tan ^{2} \beta}{\tan ^{2} \beta-1}-\frac{1}{2} M_{Z}^{2}
$$

In any case, the general situation for the soft parameters in SUGRA is to have a nonuniversal structure. In the next subsections we will analyze all these possibilities. In addition, we will also consider the possibility of relaxing the GUT scale, and study the case of an intermediate scale.

\section{1 mSUGRA scenario with a GUT scale}

As is well known, in the mSUGRA scenario with a GUT scale the lightest neutralino $\tilde{\chi}_{1}^{0}$ is mainly bino and, as a consequence, the predicted neutralino-proton cross section $\sigma_{\tilde{\chi}_{1}^{0}-p}$ is well below the accessible experimental regions for low and moderate values of $\tan \beta$ [面]. Let us recall that $\mu^{2}$ given by Eq. ( $\left.\overline{2}_{2} . \bar{l}_{1}^{\prime}\right)$, for reasonable values of $\tan \beta$, can be approximated as $\mu^{2} \approx-m_{H_{u}}^{2}-\frac{1}{2} M_{Z}^{2}$. Thus it becomes large since $m_{H_{u}}^{2}$ evolves towards large and negative values with the scale. In particular, $|\mu|$ becomes much larger than $M_{1}$ and $M_{2}$. As can be easily understood from the neutralino mass matrix, the lightest neutralino will then be mainly gaugino, and in particular bino, since at low energy $M_{1}=\frac{5}{3} \tan ^{2} \theta_{W} M_{2} \approx 0.5 M_{2}$. Now, the scattering channels through Higgs exchange are not so important. In addition, the (tree-level) mass of the CP-odd Higgs $A, m_{A}^{2}=m_{H_{d}}^{2}+m_{H_{u}}^{2}+2 \mu^{2}$, will be large because 


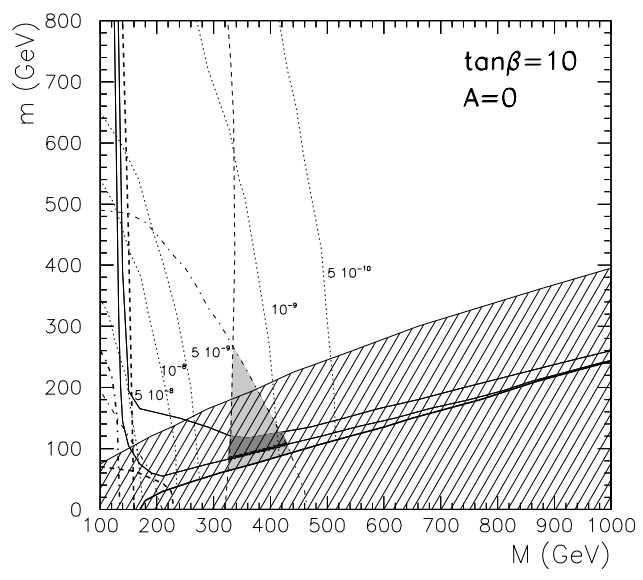

Figure 2: Scalar neutralino-proton cross section $\sigma_{\tilde{\chi}_{1}^{0}-p}$ in the parameter space of the mSUGRA scenario $(m, M)$ for $\tan \beta=10, A=0$ and $\mu>0$. The dotted curves are contours of $\sigma_{\tilde{\chi}_{1}^{0}-p}$. The region to the left of the near-vertical dashed line is excluded by the lower bound on the Higgs mass $m_{h}>114.1 \mathrm{GeV}$. The region to the left of the near-vertical double dashed line is excluded by the lower bound on the chargino mass $m_{\tilde{\chi}_{1}^{ \pm}}>103 \mathrm{GeV}$. The corner in the lower left shown also by a double dashed line is excluded by the LEP bound on the stau mass $m_{\tilde{\tau}_{1}}>87 \mathrm{GeV}$. The region bounded by dot-dashed lines is allowed by $g_{\mu}-2$. The region to the left of the double dot-dashed line is excluded by $b \rightarrow s \gamma$. From bottom to top, the solid lines are the upper bounds of the areas such as $m_{\tilde{\tau}_{1}}<m_{\tilde{\chi}_{1}^{0}}$ (double solid), $\Omega_{\tilde{\chi}_{1}^{0}} h^{2}<0.1$ and $\Omega_{\tilde{\chi}_{1}^{0}} h^{2}<0.3$. The light shaded area is favored by all the phenomenological constraints, while the dark one fulfills in addition $0.1 \leq \Omega_{\tilde{\chi}_{1}} h^{2} \leq 0.3$ (the black region on top of this indicates the WMAP range $0.094<\Omega_{\tilde{\chi}_{1}^{0}} h^{2}<0.129$ ). The ruled region is excluded because of the charge and colour breaking constraint UFB-3.

$\mu^{2}$ is large. Since the heaviest CP-even Higgs, $H$, is almost degenerate in mass with this, $m_{H}$ will also be large producing a further suppression in the scattering channels.

This general fact is shown with an example in Fig. parameter space $(m, M)$ for $\tan \beta=10, A=0$ and $\mu>0$ are plotted ${ }^{2}$. As we can see in the figure, the experimental bounds mentioned in the Introduction are very important and exclude large regions of the parameter space. This is mainly due to the combination of the Higgs mass bound with the $g_{\mu}-2$ lower bound ${ }^{3}$. The light shaded area in the figure shows the region allowed by the experimental bounds. There, the lower contour (double solid line) is obtained including also the constraint coming from the bound, $m_{\tilde{\chi}_{1}^{0}}<m_{\tilde{\tau}_{1}}$, in order to have an electrically neutral LSP. For this area $\sigma_{\tilde{\chi}_{1}^{0}-p} \approx 10^{-9} \mathrm{pb}$.

On the other hand, when the astrophysical bounds $0.1 \lesssim \Omega_{\tilde{\chi}_{1}^{0}} h^{2} \lesssim 0.3$ are also imposed the allowed area becomes very small. Only the beginning of the tail where the LSP is almost degenerate with the stau, producing efficient coannihilations, is rescued. In addition, the

\footnotetext{
${ }^{2}$ From now on we will always show throughout the paper figures taken from Ref. 逗 since they include the charge and colour breaking constraints.

${ }^{3}$ Note that we are using only the limit based on $e^{+} e^{-}$analysis, $11.3 \times 10^{-10} \leq a_{\mu}(S U G R A) \leq 56.1 \times$ $10^{-10}$, otherwise the allowed region would extend towards the right hand side of the figure.
} 

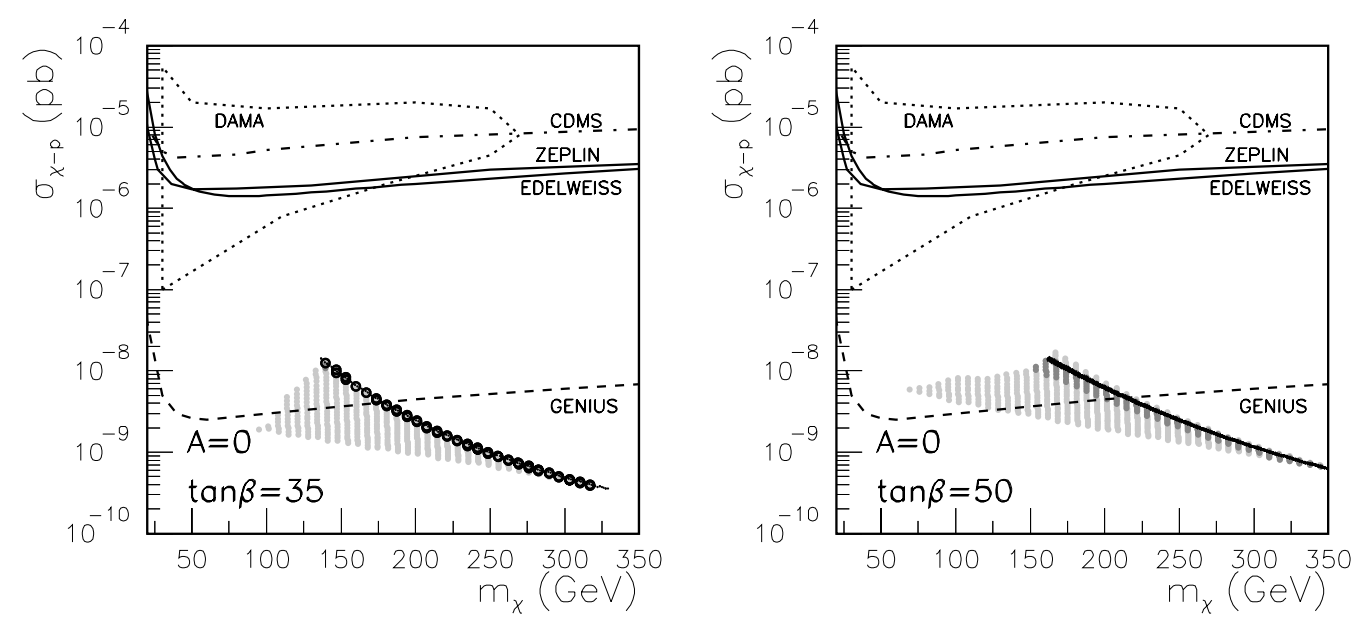

Figure 3: Scatter plot of the scalar neutralino-proton cross section $\sigma_{\tilde{\chi}_{1}^{0}-p}$ as a function of the neutralino mass $m_{\tilde{\chi}_{1}^{0}}$ in the mSUGRA scenario, for $\tan \beta=35$ and $50, A=0$ and $\mu>0$. The light grey dots correspond to points fulfilling all experimental constraints. The dark grey dots correspond to points fulfilling in addition $0.1 \leq \Omega_{\tilde{\chi}_{1}^{0}} h^{2} \leq 0.3$ (the black dots on top of these indicate those fulfilling the WMAP range $0.094<\Omega_{\tilde{\chi}_{1}^{0}} h^{2}<0.129$ ). The circles indicate regions excluded by the UFB-3 constraint. The lines corresponding to the different experiments are as in Fig.

restrictions coming from the charge and colour breaking constraints, in particular the UFB3 one, exclude also this area.

Let us recall that the UFB-3 direction, which involves the scalar fields $\left\{H_{u}, \nu_{L_{i}}, e_{L_{j}}, e_{R_{j}}\right\}$ with $i \neq j$ and thus leads also to electric charge breaking, yields the strongest bound among all the charge and colour breaking constraints. This is because the value of the potential along the UFB-3 direction is given generically by

$$
V_{\mathrm{UFB}-3}=\left(m_{H_{u}}^{2}+m_{L_{i}}^{2}\right)\left|H_{u}\right|^{2}+\frac{|\mu|}{\lambda_{e_{j}}}\left(m_{L_{j}}^{2}+m_{e_{j}}^{2}+m_{L_{i}}^{2}\right)\left|H_{u}\right|-\frac{2 m_{L_{i}}^{4}}{g^{\prime 2}+g_{2}^{2}},
$$

and then it may become more negative than the MSSM potential at the minimum, when $m_{H_{u}}^{2}$ evolves towards negative values.

In conclusion, the results indicate that the whole parameter space for $\tan \beta=10$ is excluded on these grounds. In fact, it is possible to show that $\tan \beta \lesssim 20$ is excluded for any value of $A$ [囟.

The neutralino-proton cross section can be increased when the value of $\tan \beta$ is increased [5] cross section is proportional to $1 / \cos \beta$. In addition, the bottom Yukawa coupling increases, and as a consequence $m_{H_{d}}^{2}$ decreases, implying that $m_{A}^{2}=m_{H_{d}}^{2}+m_{H_{u}}^{2}+2 \mu^{2}$ also decreases. Since, as mentioned above, $m_{H} \approx m_{A}$, this will also decrease significantly. Indeed, scattering channels through Higgs exchange are more important now and their contributions to the cross section will increase it. Thus, in principle, we can even enter in the DAMA region. However, the present experimental constraints exclude this possibility

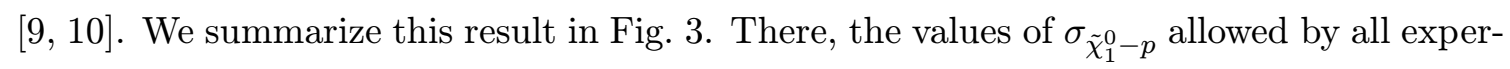


imental constraints, as a function of the neutralino mass $m_{\tilde{\chi}_{1}^{0}}$, are shown for $\tan \beta=35,50$ and $A=0$. Dark grey dots correspond to those points having a relic neutralino density within the preferred range $0.1 \leq \Omega h^{2} \leq 0.3$. Given the narrow range of these points for the case $\tan \beta=35$, they overlap in the figure with those excluded by the UFB-3 constraint (shown with circles). We observe that, generically, the cross section and the neutralino mass are constrained to be (for any value of $A$ ) $5 \times 10^{-10} \lesssim \sigma_{\tilde{\chi}_{1}^{0}-p} \lesssim 3 \times 10^{-8}$ pb and $120 \lesssim m_{\tilde{\chi}_{1}^{0}} \lesssim 320 \mathrm{GeV}$, respectively.

It is possible to show that qualitatively similar results are obtained when dark matter is analyzed in the so-called focus-point supersymmetry scenario [i] $\left.]_{-1}^{1}\right]$.

Obviously, given the above results, $\sigma_{\tilde{\chi}_{1}^{0}-p} \lesssim 3 \times 10^{-8} \mathrm{pb}$, in the mSUGRA scenario with a GUT scale more sensitive detectors producing further data are needed. As discussed in the Introduction, many dark matter detectors are being projected. Particularly interesting is the case of GENIUS, where values of the cross section as low as $\approx 10^{-9}$ pb will be accessible, although this might not be sufficient depending on the values of the parameters (see Fig. 'i $3 \overline{3}$ ).

\section{2 mSUGRA scenario with an intermediate scale}

The analysis of the cross section $\sigma_{\tilde{\chi}_{1}^{0}-p}$ carried out above in the context of mSUGRA, was performed assuming the unification scale $M_{G U T} \approx 10^{16} \mathrm{GeV}$. However, there are several interesting phenomenological arguments in favour of SUGRA scenarios with scales $M_{I} \approx 10^{10-14} \mathrm{GeV}$, such as to explain neutrino masses, the scale of axion physics, and others (see e.g. the discussion in Ref. [1] 120 ] and references therein). In addition, the string scale may be anywhere between the weak and the Planck scale, and explicit scenarios with intermediate scales may arise in the context of D-brane constructions from type I strings, as we will discuss in Section 3. Inspired by all these scenarios, to use the value of the initial scale $M_{I}$ as a free parameter for the running of the soft terms is particularly interesting.

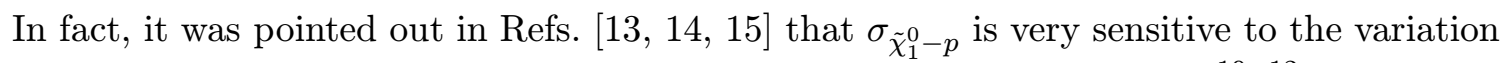
of $M_{I}$ for the running of the soft terms. For instance, by taking $M_{I}=10^{10-12} \mathrm{GeV}$ rather than $M_{G U T}$, regions in the parameter space of mSUGRA can be found where $\sigma_{\tilde{\chi}_{1}^{0}-p}$ is two orders of magnitude larger than for $M_{G U T}$ [1순,

The fact that smaller scales imply a larger $\sigma_{\tilde{\chi}_{1}^{0}-p}$ can be explained with the variation in the value of $\mu$ with $M_{I}$. One observes that, for $\tan \beta$ fixed, the smaller the initial scale for the running the smaller the numerator in the first piece of Eq. (2.2. $\left.\overline{1}^{\prime}\right)$ becomes. This can easily be understood from the evolution of $m_{H_{u}}^{2}$ with the scale. Clearly, when the value of the initial scale is reduced the RGE running is shorter and, as a consequence, the negative contribution $m_{H_{u}}^{2}$ to $\mu^{2}$ in Eq. $\left(\overline{2}_{2}^{2} . \overline{1}_{1}^{1}\right)$ becomes less important. Then, $|\mu|$ decreases and therefore the Higgsino composition of the lightest neutralino increases. Eventually, $|\mu|$ will be of the order of $M_{1}, M_{2}$ and $\tilde{\chi}_{1}^{0}$ will be a mixed Higgsino-gaugino state. In addition, when $|\mu|$ decreases $m_{A}^{2}=m_{H_{d}}^{2}+m_{H_{u}}^{2}+2 \mu^{2}$ also decreases. As mentioned in the previous Subsection when talking about increasing $\tan \beta, H$ will decrease and therefore the scattering channels through Higgs exchange will increase the cross section. 

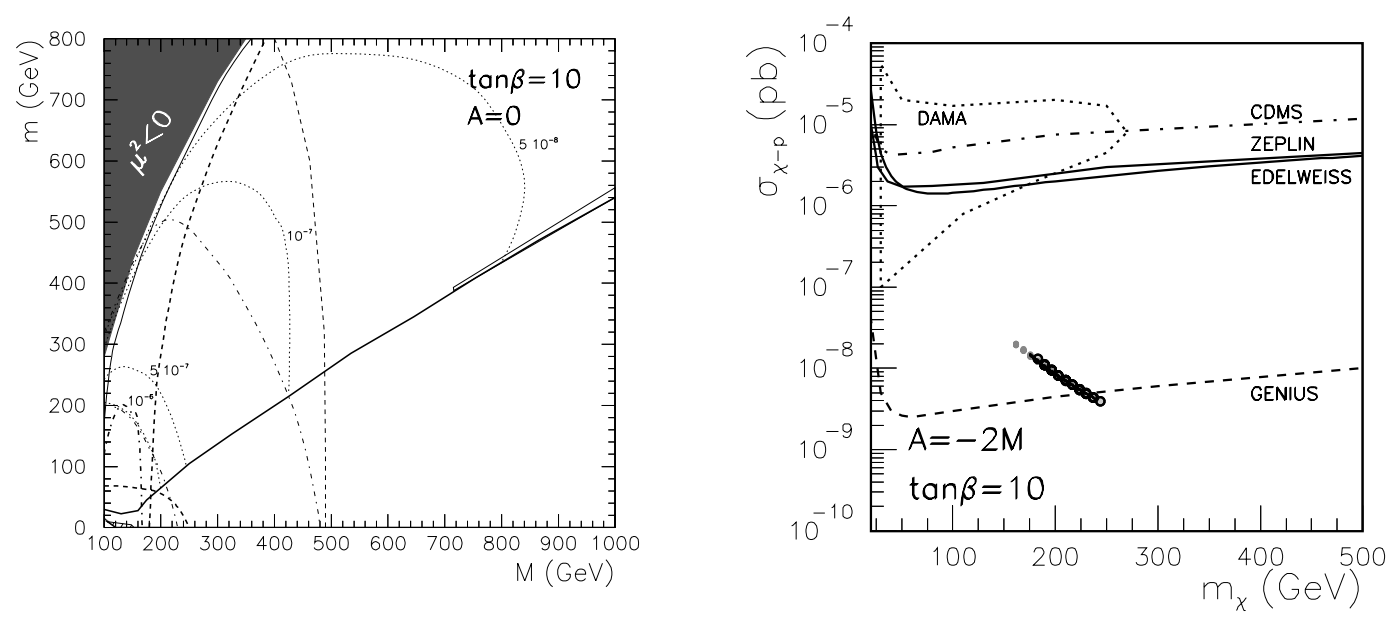

Figure 4: Left: The same as in Fig. 产 but for the intermediate scale $M_{I}=10^{11}$. The black area is excluded because $\mu^{2}$ becomes negative. The white region at the bottom bounded by a solid line is excluded because $m_{\tilde{\tau}_{1}}^{2}$ becomes negative. Right: The same as in Fig. $\bar{\beta}_{1}^{\prime}$ but for the intermediate scale $M_{I}=10^{11} \mathrm{GeV}$, with $\tan \beta=10$ and $A=-2 M$.

In the plot on the left frame of Fig. 崔 we show the result for $M_{I}=10^{11} \mathrm{GeV}$, with $\tan \beta=10$ and $A=0$. This case can be compared with the one in Fig. $\stackrel{\overline{2}}{2}$, where $M_{G U T}$ is used. Clearly, for the same values of the parameters, larger cross sections can be obtained with the intermediate scale. It is worth noticing that even with this moderate value of $\tan \beta, \tan \beta=10$, there are regions where the cross section enters in the DAMA area, $\sigma_{\tilde{\chi}_{1}^{0}-p} \approx 10^{-6} \mathrm{pb}$. However, at the end of the day, the whole parameter space is forbidden due to the combination of the Higgs mass bound with the $g_{\mu}-2$ lower bound. Notice also that $\Omega_{\tilde{\chi}_{1}^{0}} h^{2}$ is smaller than 0.1 in most of the parameter space. Only tiny regions bounded by solid lines in the figure, and therefore with $0.1 \leq \Omega_{\tilde{\chi}_{1}^{0}} h^{2} \leq 0.3$, can be found. For other values of $A$, such as $A=-2 M$, there are small regions where the $m_{h}$ and $g_{\mu}-2$ bounds are compatible. Also, larger regions with $0.1 \leq \Omega_{\tilde{\chi}_{1}^{0}} h^{2} \leq 0.3$ are present. In any case, finally the lower bound for $m_{h}$ implies that the allowed cross sections do not enter in the DAMA area.

One also finds that the regions excluded by the UFB-3 constraint are much smaller than in those cases where the initial scale is the GUT one. For example for $A=0$ no region is excluded (see however Fig. ${ }_{2}^{2}$ i for the GUT case).

In the plot on the right frame of Fig. 峷 we summarize the above results for $\tan \beta=$ 10 , concerning the cross section, showing the values of $\sigma_{\tilde{\chi}_{1}^{0}-p}$ allowed by all experimental constraints as a function of the neutralino mass $m_{\tilde{\chi}_{1}^{0}}$, for $A=-2 M$. Only in this case there are dark grey dots corresponding to points having a relic neutralino density within the preferred range $0.1 \leq \Omega_{\tilde{\chi}_{1}^{0}} h^{2} \leq 0.3$. Given the narrow range of these points, they overlap in the figure with those excluded by the UFB-3 constraint.

Qualitatively, similar results are obtained for larger values of $\tan \beta$. For example, for $\tan \beta=35$ only for $A=-2 M$ we obtain points allowed by all experimental and 

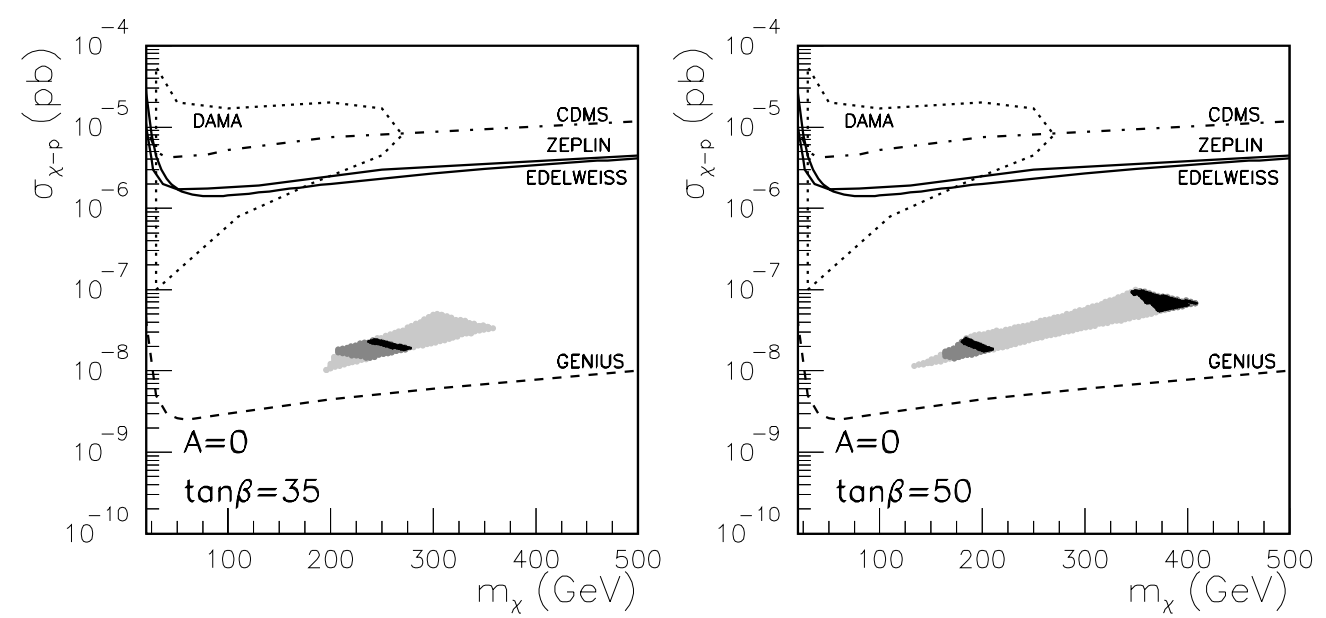

Figure 5: The same as in Fig. $\overline{3}_{1}$ but for the case discussed in Eq. (2.2.3i) with non-universal soft gaugino masses, $\delta_{1,2}^{\prime}=0, \delta_{3}^{\prime}=-0.5$.

astrophysical constraints. For these, $\sigma_{\tilde{\chi}_{1}^{0}-p} \lesssim 10^{-8} \mathrm{pb}$. Let us finally mention that in the case of $\tan \beta=50$, for $A=-M$ there are points allowed by all experimental and astrophysical constraints with $\sigma_{\tilde{\chi}_{1}^{0}-p} \lesssim 10^{-7} \mathrm{pb}$.

Summarizing, when an intermediate scale is considered in mSUGRA, although the cross section increases significantly the experimental bounds impose $\sigma_{\tilde{\chi}_{1}^{0}-p} \lesssim 4 \times 10^{-7} \mathrm{pb}$. And, in fact, at the end of the day, the preferred astrophysical range for the relic neutralino density, $0.1 \leq \Omega_{\tilde{\chi}_{1}^{0}} h^{2} \leq 0.3$, imposes $\sigma_{\tilde{\chi}_{1}^{0}-p} \lesssim 10^{-7}$ pb. Clearly, present experiments are not still sufficient, and more sensitive detectors producing further data are needed, as in the case of a GUT scale.

\subsection{SUGRA scenario with non-universal gaugino masses}

The general situation for the soft parameters in SUGRA is to have a non-universal structure. For the case of the gaugino masses this is due to the non-universality of the gauge kinetic functions associated to the different gauge groups $f_{a}\left(h_{m}\right)$. For example, non-universal gaugino masses are obtained if the $f_{a}$ have a different dependence on the hidden sector fields $h_{m}$ breaking SUSY.

Analyses of the neutralino-proton cross section using $S U(5)$ GUT relations for gaugino

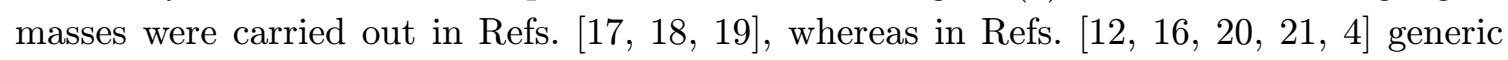
non-universal soft masses were used. It was also realized that the non-universality in the gaugino masses can increase the cross section.

Let us parameterize this non-universality at $M_{G U T}$ as follows:

$$
M_{1}=M\left(1+\delta_{1}^{\prime}\right), \quad M_{2}=M\left(1+\delta_{2}^{\prime}\right), \quad M_{3}=M\left(1+\delta_{3}^{\prime}\right),
$$

where $M_{1,2,3}$ are the bino, wino and gluino masses, respectively. Let us discuss now which values of the parameters are interesting in order to increase the cross section with respect to the universal case $\delta_{i}^{\prime}=0$. In this sense, it is worth noticing that $M_{3}$ appears in the 
RGEs of squark masses, so e.g. their contribution proportional to the top Yukawa coupling in the RGE of $m_{H_{u}}^{2}$ will do this less negative if $M_{3}$ is small, and therefore $\mu^{2}$ will become smaller in this case. However, small values of $M_{3}$ also lead to an important decrease in the Higgs mass. In addition, $b \rightarrow s \gamma$ and $g_{\mu}-2$ constraints are also relevant.

Summarizing, although the cross section increases with respect to the universal case, the present experimental constraints exclude points entering in the DAMA region. This is shown in Fig. $\underline{\underline{S}}_{1}^{\prime}$ for $\tan \beta=35,50$ and $A=0$, using $\delta_{1,2}^{\prime}=0, \delta_{3}^{\prime}=-0.5$, where one can see that there are points allowed by all experimental and astrophysical constraints, but they correspond to $\sigma_{\tilde{\chi}_{1}^{0}-p} \lesssim 10^{-7} \mathrm{pb}$.

Finally, let us remark that increasing the cross section through values at low energy of $m_{H_{u}}^{2}$ less negatives implies less important UFB constraints. Now these are not very relevant.

\subsection{SUGRA scenario with non-universal scalar masses}

As mentioned in the previous subsection, the general situation for the soft parameters in SUGRA is to have a non-universal structure. For the case of the observable scalar masses this is due to the non-universal couplings in the Kähler potential between the hidden sector fields breaking SUSY and the observable sector fields. For example, $K=$ $\sum_{\alpha} \tilde{K}_{\alpha}\left(h_{m}, h_{m}^{*}\right) C_{\alpha} C_{\alpha}^{*}$, with $\tilde{K}_{\alpha}$ a function of the hidden-sector fields $h_{m}$, will produce non-universal scalar masses $m_{\alpha} \neq m_{\beta}$ if $\tilde{K}_{\alpha} \neq \tilde{K}_{\beta}$.

Analyses of the dark matter cross section using generic non-universal soft masses were

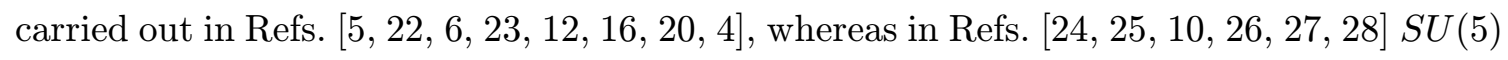
or $S O(10)$ GUT relations were used. In the light of the recent experimental results, one important consequence of the non-universality is that the cross section can be increased in some regions of the parameter space

Let us then parameterized the non-universality in the Higgs sector as follows:

$$
m_{H_{d}}^{2}=m^{2}\left(1+\delta_{1}\right), \quad m_{H_{u}}^{2}=m^{2}\left(1+\delta_{2}\right) .
$$

In fact the non-universalities in this sector give the most important effect, and including the ones in the sfermion sector the cross section only increases slightly. Thus in what follows we will only consider $\delta_{1,2} \neq 0$.

As discussed for intermediate scales in Subsection 2.2, an important factor in order to increase the cross section consists in reducing the value of $|\mu|$. This value is determined by condition $\mu^{2} \approx-m_{H_{u}}^{2}-\frac{1}{2} M_{Z}^{2}$ and can be significantly reduced for some choices of the $\delta$ 's. We can have a qualitative understanding of the effects of the $\delta$ 's on $\mu$ from the following. When $m_{H_{u}}^{2}$ at $M_{G U T}$ increases, i.e. choosing $\delta_{2}>0$, its negative low-energy contribution to the above condition becomes less important.

In addition, there is another relevant way of increasing the cross section using the non-universalities of the Higgs sector. Note that decreasing $m_{H_{d}}^{2}$, i.e. choosing $\delta_{1}<0$, leads to a decrease in $m_{A}^{2}=m_{H_{d}}^{2}+m_{H_{u}}^{2}+2 \mu^{2}$, and therefore in the mass of the heaviest Higgs $H$. This produces naturally an increase in the cross section ${ }^{4}$.

\footnotetext{
${ }^{4}$ This effect might also be important when non-universal gaugino masses are taken into account. The
} 

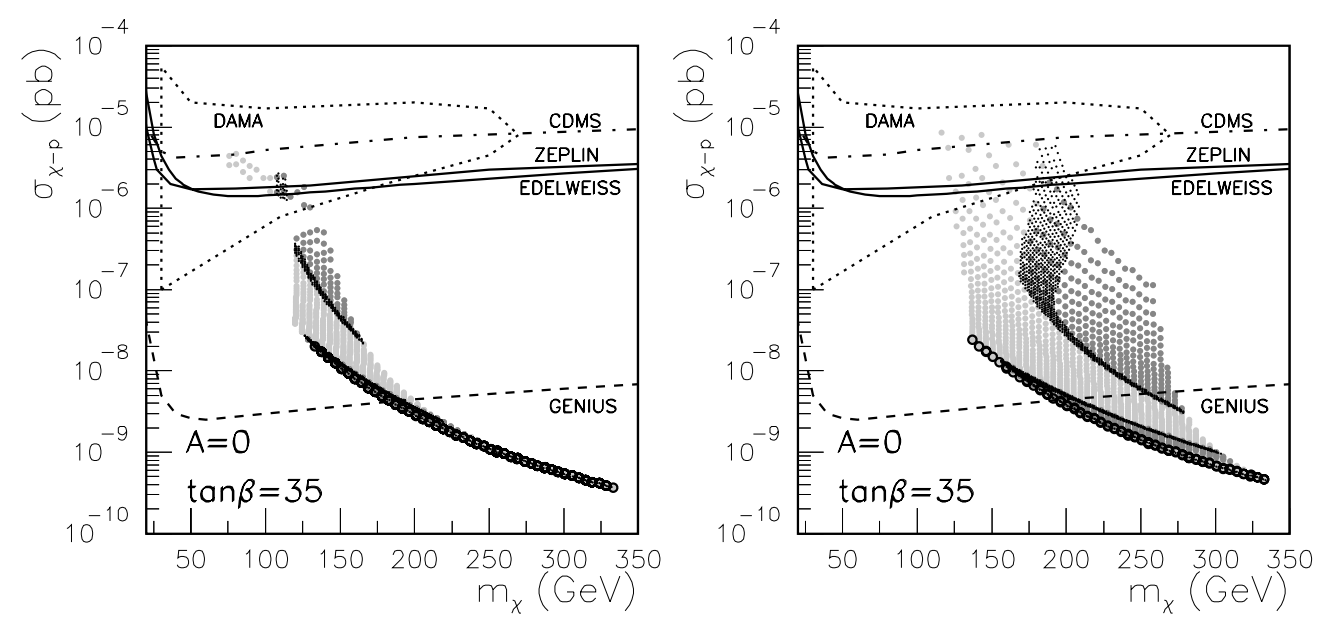

Figure 6: The same as in Fig. $\underline{3}_{1}$ but for the non-universal cases $\left.b\right) \delta_{1}=-1, \delta_{2}=0$ (left), and c) $\delta_{1}=-1, \delta_{2}=1$ (right), discussed in Eq. (20.5), with $\tan \beta=35$ and $A=0$.

Thus we will see that, unlike the previous scenarios, with non-universalities in the scalar sector is possible to obtain large values of the cross section, and even some points enter in the DAMA area fulfilling all constraints. Let us analyze three representative cases with [4]

$$
\begin{aligned}
& \text { a) } \delta_{1}=0 \quad, \quad \delta_{2}=1, \\
& \text { b) } \delta_{1}=-1, \quad \delta_{2}=0 \\
& \text { c) } \delta_{1}=-1, \delta_{2}=1
\end{aligned}
$$

Clearly, the above discussion about decreasing $\mu^{2}$ applies well to case a), where the variation in $m_{H_{u}}^{2}$ through $\delta_{2}$ is relevant. For $\tan \beta=35$, although the cross section increases with respect to the universal case, and is generically above the GENIUS lower limit, the present experimental constraints exclude points entering in the DAMA area. We need to go to $\tan \beta=50$ in order to find points entering in the DAMA area, and fulfilling the astrophysical bounds.

For case $b$ ) the cross section increases also substantially with respect to the universal case. Now $\delta_{2}$ is taken vanishing and therefore the value of $\mu$ is essentially not modified with respect to the universal case. However, taking $\delta_{1}=-1$ produces an increase in the cross section through the decrease in $m_{A}^{2}$, as discussed previously. As shown explicitly in in the plot on the left frame of Fig. $\overline{6}$, for $\tan \beta=35$ and $A=0$, there are points in the DAMA region. For $\tan \beta=50$, similarly to case $a$ ), there are points entering in the DAMA area, and part of them fulfil the astrophysical bounds.

Finally, given the above situation concerning the enhancement of the neutralino-proton cross section for $a$ ) and $b$ ), it is clear that the combination of both cases might be interesting. This is carried out in case $c$ ) where we take $\delta_{1}=-1$ and $\delta_{2}=1$. As shown in the plot on

contribution of $M_{3}$ proportional to the bottom Yukawa coupling in the RGE of $m_{H_{d}}^{2}$ will do this smaller if $M_{3}$ is large $\left[29_{1}^{2}\right]$. 


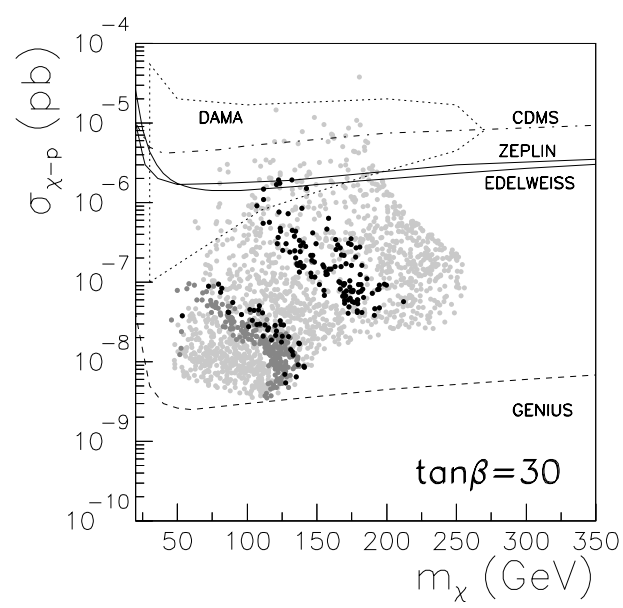

Figure 7: The same as in Fig. 这 but for a D-brane scenario with string scale $M_{I}=10^{12} \mathrm{GeV}$, and $\tan \beta=30$.

the right frame of Fig. $\overline{6}_{1}^{1}$, cross sections as large as $\sigma_{\tilde{\chi}_{1}^{0}-p} \gtrsim 10^{-6} \mathrm{pb}$, entering in DAMA and fulfilling all experimental and astrophysical bounds, can be obtained for $\tan \beta=35$ and $A=0$. This is also the case for $\tan \beta=50$.

In summary, when non-universal scalars are allowed in SUGRA, for some special choices of the non-universality, the cross section can be increased a lot with respect to the universal scenario. It is even possible, for some particular values of the parameters, to find points allowed by all experimental and astrophysical constraints with $\sigma_{\tilde{\chi}_{1}^{0}-p} \approx 10^{-6}$ $\mathrm{pb}$, and therefore inside the DAMA area ${ }^{5}$. Note however that these points would be basically excluded by the other underground experiments. In any case, the interesting result is that large regions accessible for future experiments are present.

\section{Superstring scenarios}

D-brane constructions are explicit scenarios where two of the interesting situations studied in the previous Section, non-universality and intermediate scales, may occur. Concerning the latter, it was recently realized that the string scale may be anywhere between the weak and the Plank scale (see e.g. the discussion in Ref. [1] fact scenarios with the gauge group and particle content of the SUSY standard model lead naturally to intermediate values for the string scale, in order to reproduce the value of gauge couplings deduced from experiments [i] $\left[\begin{array}{l}1 \\ 5\end{array}\right]$. In addition, the soft terms turn out to be generically non universal.

Due to these results, in principle, large cross sections might be obtained. A recent re-analysis allows us to think that this is the case. Very preliminary results are shown in Fig. $\bar{T}_{1}$, where one can see that even points entering in DAMA are allowed [20 $\left.2 \overline{9}\right]$ ].

\footnotetext{
${ }^{5}$ This is similar to what occurs in the so-called effMSSM scenario, where the parameters are defined directly at the electroweak scale [30.
} 


\section{Conclusions}

We have carried out a theoretical analysis of the possibility of detecting dark matter directly in current and projected experiments. In particular, we have studied the value of the neutralino-nucleon cross section in several supergravity and superstring scenarios. In addition to the usual experimental and astrophysical constraints we have imposed on the parameter space the absence of dangerous charge and colour breaking minima.

In the usual mSUGRA scenario, where the soft terms are assumed to be universal, and the GUT scale is considered, the cross section is constrained to be $\sigma_{\tilde{\chi}_{1}^{0}-p} \lesssim 3 \times 10^{-8}$ pb. Obviously, in this case, present experiments are not sufficient and more sensitive detectors producing further data are needed. A similar conclusion is obtained when an intermediate scale is considered. Although the cross section increases significantly, the experimental bounds impose $\sigma_{\tilde{\chi}_{1}^{0}-p} \lesssim 4 \times 10^{-7} \mathrm{pb}$. And, in fact, at the end of the day, the preferred astrophysical range for the relic neutralino density, $0.1 \leq \Omega_{\tilde{\chi}_{1}^{0}} h^{2} \leq 0.3$, imposes $\sigma_{\tilde{\chi}_{1}^{0}-p} \lesssim 10^{-7} \mathrm{pb}$. Still present experiments are not sufficient.

When non-universal scalars are allowed in SUGRA, for some special choices of the nonuniversality, the cross section can be increased a lot with respect to the universal scenario. It is even possible, for some particular values of the parameters, to find points allowed by all experimental and astrophysical constraints with $\sigma_{\tilde{\chi}_{1}^{0}-p} \approx 10^{-6} \mathrm{pb}$, and therefore inside the DAMA area. For non-universal gauginos, although the cross section increases, the experimental bounds exclude this possibility.

On the other hand, the low-energy limit of superstring theory is SUGRA, and therefore the neutralino will also be a candidate for dark matter in these scenarios. In this context we have studied D-branes configurations from type I string, where intermediate scales and non-universal soft terms arise naturally. A preliminary analysis allows us to think that some regions of the parameter space may have large values of the cross section, even compatible with DAMA.

Finally, it is worth recalling that although the DAMA result is controversial because the negative search result obtained by other recent experiments, our main point is that large regions of the parameter space accessible for experiments are present in several SUSY scenarios.

\section{Acknowledgements}

We thank D.G. Cerdeño, E. Gabrielli and M.E. Gomez as co-authors of the works reported in this talk.

\section{References}

[1] For a recent review see, C. Muñoz, 'Dark matter detection in the light of recent experimental results', hep-ph/0309346.

[2] P. Belli, R. Cerulli, N. Fornengo and S. Scopel, Phys. Rev. D66 (2002) 043503.

[3] C.J. Copi and L.M. Krauss, Phys. Rev. D67 (2003) 103507.

[4] D.G. Cerdeño, E. Gabrielli, M.E. Gómez and C. Muñoz, J. High Energy Phys. 06 (2003) 030. 
[5] A. Bottino, F. Donato, N. Fornengo and S. Scopel, Phys. Rev. D59 (1999) 095004.

[6] E. Accomando, R. Arnowitt, B. Dutta and Y. Santoso, Nucl. Phys. B585 (2000) 124.

[7] M.E. Gomez and J.D. Vergados, Phys. Lett. B512 (2001) 252; hep-ph/0105115.

[8] J. Ellis, T. Falk, G. Ganis, K.A. Olive and M. Srednicki, Phys. Lett. B510 (2001) 236.

[9] J. Ellis, A. Ferstl and K.A. Olive, Phys. Lett. B532 (2002) 318.

[10] R. Arnowitt and B. Dutta, hep-ph/0112157.

[11] J.L. Feng, K.T. Matchev and F. Wilczek, Phys. Lett. B482 (2000) 388; H. Baer and C. Balazs, JCAP 05 (2003) 006.

[12] D.G. Cerdeño, S. Khalil and C. Muñoz, hep-ph/0105180.

[13] E. Gabrielli, S. Khalil, C. Muñoz and E. Torrente-Lujan Phys. Rev. D63 (2001) 025008.

[14] D. Bailin, G.V. Kraniotis and A. Love, Phys. Lett. B491 (2000) 161.

[15] D.G. Cerdeño, E. Gabrielli, S. Khalil, C. Muñoz and E. Torrente-Lujan, Nucl. Phys. B603 (2001) 231.

[16] D.G. Cerdeño, E. Gabrielli and C. Muñoz, hep-ph/0204271.

[17] A. Corsetti and P. Nath, Phys. Rev. D64 (2001) 125010.

[18] V. Bertin, E. Nezri and J. Orloff, J. High Energy Phys. 02 (2003) 046.

[19] U. Chattopadhyay and D.P. Roy, hep-ph/0304108.

[20] R. Arnowitt and B. Dutta, hep-ph/0210339.

[21] A. Birkedal-Hansen and B.D. Nelson, Phys. Rev. D67 (2003) 095006.

[22] R. Arnowitt and P. Nath, Phys. Rev. D60 (1999) 044002.

[23] See J. Ellis, A. Ferstl, K.A. Olive and Y. Santoso, Phys. Rev. D67 (2003) 123502, and references therein.

[24] M. Drees, Y.G. Kim, T. Kobayashi and M.M. Nojiri, Phys. Rev. D63 (2001) 115009.

[25] Y.G. Kim and M.M. Nojiri, Prog. Theor. Phys. 106 (2001) 561.

[26] R. Dermisek, S. Raby, L. Roszkowski, R. Ruiz De Austri, J. High Energy Phys. 04 (2003) 037.

[27] H. Baer, C. Balazs, A. Belyaev and J. O'Farril, JCAP 09 (2003) 007.

[28] See S. Profumo, J. High Energy Phys. 06 (2003) 052, and references therein.

[29] D.G. Cerdeño and C. Muñoz, in preparation.

[30] L. Bergström and P. Gondolo, Astropart. Phys. 5 (1996) 263; E.A. Baltz and P. Gondolo, Phys. Rev. D67 (2003) 063503.

[31] See V.A. Bednyakov and H.V. Klapdor-Kleingrothaus, Phys. Rev. D63 (2001) 095005, and references therein.

[32] V. Mandic, A.T. Pierce, P. Gondolo and H. Murayama hep-ph/0008022; V.A.Bednyakov, Phys. Atom. Nucl. 66 (2003) 490.

[33] See A. Bottino, F. Donato, N. Fornengo and S. Scopel, Phys. Rev. D68 (2003) 043506, and references therein.

[34] Y.G. Kim, T. Nihei, L. Roszkowski and R. Ruiz de Austri, J. High Energy Phys. 12 (2002) 034 . 